Volume 25 (2019) 224-231

DOI: $10.24330 /$ ieja.504159

\title{
GROUP PARTITIONS VIA COMMUTATIVITY
}

\author{
A. Mahmoudifar, A. R. Moghaddamfar and F. Salehzadeh \\ Received: 1 August 2018; Revised: 19 September 2018; Accepted: 24 October 2018 \\ Communicated by Arturo Magidin
}

\begin{abstract}
Let $G$ be a nonabelian group, $A \subset G$ an abelian subgroup and $n \geqslant 2$ an integer. We say that $G$ has an $n$-abelian partition with respect to $A$, if there exists a partition of $G$ into $A$ and $n$ disjoint commuting subsets $A_{1}, A_{2}, \ldots, A_{n}$ of $G$, such that $\left|A_{i}\right|>1$ for each $i=1,2, \ldots, n$. We classify all nonabelian groups, up to isomorphism, which have an $n$-abelian partition, for $n=2$ and 3 .
\end{abstract}

Mathematics Subject Classification (2010): 05C25, 20D06

Keywords: $n$-Abelian partition, commuting graph

\section{Introduction and preliminaries}

Let $\Gamma$ be a simple graph and $m, n$ two non-negative integers. We say that $\Gamma$ is $(m, n)$-partitionable if its vertex set can be partitioned into $m$ independent sets $I_{1}, \ldots, I_{m}$ and $n$ cliques $C_{1}, \ldots, C_{n}$; that is

$$
V_{\Gamma}=I_{1} \uplus I_{2} \uplus \cdots \uplus I_{m} \uplus C_{1} \uplus C_{2} \uplus \cdots \uplus C_{n} .
$$

Such a partition of $V_{\Gamma}$ is called a $(m, n)$-partition of $\Gamma$ (see [3]). We shall note some special cases: (1,1)-partitionable graphs are called split graphs (see [6]), (1,0)partitionable graphs are called edgeless graphs, $(0,1)$-partitionable graphs are called complete graphs. In particular, in the case when $m=0$ or $n=0$, we essentially split $\Gamma$ into $n$ cliques,

$$
V_{\Gamma}=C_{1} \uplus C_{2} \uplus \cdots \uplus C_{n},
$$

or $m$ independent sets,

$$
V_{\Gamma}=I_{1} \uplus I_{2} \uplus \cdots \uplus I_{m},
$$

respectively.

In the following all groups will be assumed finite. We now focus our attention on a graph associated with a finite group - the so-called commuting graph. Let $G$ be a finite group and $X$ a nonempty subset of $G$. The commuting graph $\mathcal{C}(G, X)=\mathcal{C}(X)$, has $X$ as its vertex set with two distinct elements of $X$ joined by an edge when they commute in $G$. Commuting graphs have been investigated by many authors in 
various contexts, see for instance $[4,5,8]$. Clearly, $\mathcal{C}(G)$ is $(0, n)$-partitionable if and only if $G$ can be partitioned into $n$ commuting subsets. This suggests the following definition.

Definition 1.1. Let $G$ be a nonabelian group and $A \subset G$ a commuting subset containing the identity element. We say that $G$ has an $n$-abelian partition (with respect to $A$ ), if there exists a partition of $G$ into $A$ and $n$ disjoint commuting subsets $A_{1}, A_{2}, \ldots, A_{n}$ of $G, G=A \uplus A_{1} \uplus A_{2} \uplus \cdots \uplus A_{n}$, such that $\left|A_{i}\right|>1$ for each $i=1,2, \ldots, n$.

Note that, if a group $G$ has an $n$-abelian partition, then $|G| \geqslant 2 n+1$ and the commuting graph $\mathcal{C}(G)$ is a $(0, n+1)$-partitionable graph. The following elementary result shows that there does not exist a 1-abelian partition of a group with respect to a commuting subset including the identity element.

Lemma 1.2. If a nonabelian group has an n-abelian partition with respect to a commuting subset including the identity element, then $n>1$.

Proof. Suppose the contrary. Let $G=A \uplus A_{1}$ be a 1-abelian partition of $G$ with respect to a commuting subset $A$ including the identity element. Then either $|A| \geqslant|G| / 2$ or $\left|A_{1}\right| \geqslant|G| / 2$. In the first case, $\langle A\rangle=G$, and in the second case, $\left\langle A_{1}\right\rangle=G$. Thus, in both cases $G$ is abelian, a contradiction.

Clearly, if $G$ has a commuting subset $A$ (including the identity element) for which every element outside of $A$ has order larger than 2, then $G$ has an $n$-abelian partition for some integer $n \geqslant 2$. Actually, in this case, we can pair every element outside of $A$ with its inverse. Thus, for some integer $n \geqslant 2$, we have

$$
G \backslash A=\left\{x_{i}, x_{i}^{-1} \mid i=1,2, \ldots, n\right\},
$$

and so

$$
G=A \uplus\left\{x_{1}, x_{1}^{-1}\right\} \uplus\left\{x_{2}, x_{2}^{-1}\right\} \uplus \cdots \uplus\left\{x_{n}, x_{n}^{-1}\right\},
$$

would be an $n$-abelian partition of $G$. In particular, this shows that every group of odd order has an $n$-abelian partition with respect to $A=1$, where $n=(|G|-1) / 2$.

Finally, our discussion in the previous paragraph and next lemma show that the problem of finding an abelian partition of a group reduces to the case of centerless groups of even order.

Lemma 1.3. If $G$ is a nonabelian group with $|Z(G)|>1$, then $G$ has an n-abelian partition with respect to $Z(G)$, where $n=|G: Z(G)|-1$. 
Proof. Let $Z=Z(G), n=|G: Z|-1$ and $T=\left\{x_{0}, x_{1}, \ldots, x_{n}\right\}$ is a transversal for $Z$ in $G$, where $x_{0} \in Z$. Clearly, as the cosets of the centre are commuting subsets of $G$, we have the following $n$-abelian partition for $G$ with respect to $Z$ :

$$
G=Z \uplus Z x_{1} \uplus Z x_{2} \uplus \cdots \uplus Z x_{n},
$$

as required.

For example, the dihedral groups

$$
D_{2 k}=\left\langle a, b \mid a^{k}=b^{2}=1, b^{-1} a b=a^{-1}\right\rangle,
$$

where $k>2$ is an even integer, have a $(k-1)$-abelian partition with respect to $Z\left(D_{2 k}\right)=\left\{1, a^{k / 2}\right\}$. Nevertheless, the dihedral groups $D_{2 k}$, where $k>1$ is an odd integer, have no such a partition.

Lemma 1.4. For any integer $n \geqslant 2$, there exists a group $G$ which has an n-abelian partition with respect to an abelian subgroup.

Proof. It follows by considering the dihedral group $D_{2 k}$, where $k=2 n$. Take $A=\langle a\rangle$ and $A_{i}=Z a^{i} b, i=1,2, \ldots, n$, where $Z=Z\left(D_{2 k}\right)=\left\{1, a^{n}\right\}$.

We are particularly interested in groups that have an abelian partition with respect to an abelian subgroup. In fact, in all the examples we know of groups with abelian partitions, they have a partition with respect to an abelian subgroup. Therefore, we present the following conjecture for future work.

Conjecture 1.5. If $G$ is a group with an abelian partition (with respect to a commuting subset containing the identity element), then $G$ has an abelian partition with respect to an abelian subgroup.

In the next section, the structure of groups $G$ which have an $n$-abelian partition with respect to an abelian subgroup, for $n=2$ and 3, is obtained (Theorems 2.4 and 2.5).

All notation and terminology for groups are standard, however, we introduce some more notation. Following S. M. Belcastro and G. J. Sherman [1], we denote by \# Cent $(G)$ the number of distinct centralizers in a group $G$. We shall say that a group $G$ is $n$-centralizer if $\# \operatorname{Cent}(G)=n$. A noncommuting set of a group $G$ (i.e., an independent set in commuting graph $\mathcal{C}(G)$ ) has the property that no two of its elements commute under the group operation. We denote by $\operatorname{nc}(G)$ the maximum cardinality of any noncommuting set of $G$ (the independence number of $\mathcal{C}(G)$ ). Finally, the number of distinct conjugacy classes of $G$ is denoted by $\mathrm{k}(G)$. We use $\mathbb{A}_{n}$ and $\mathbb{S}_{n}$ to denote an alternating and a symmetric group of degree $n$, respectively. 


\section{Main results}

If $G$ has an $n$-abelian partition, then the pigeon-hole principle gives $\operatorname{nc}(G) \leqslant$ $n+1$. Thus, by Corollary 2.2 (a) in [2], we obtain

$$
|G| \leqslant \mathrm{nc}(G) \cdot \mathrm{k}(G) \leqslant(n+1) \mathrm{k}(G),
$$

which immediately implies that

$$
n \geqslant\left\lfloor\frac{|G|}{\mathrm{k}(G)}\right\rfloor-1
$$

Therefore, we have found a lower bound for $n$, when $\mathrm{k}(G)$ is known.

Example 2.1. Let $G=L_{2}(q)$, where $q \geqslant 4$ is a power of 2. We know that $|G|=q\left(q^{2}-1\right)$ and $\mathrm{k}\left(L_{2}(q)\right)=q+1$. Thus, if $G$ has an $n$-abelian partition, then by Eq. (1), we get $n \geqslant q^{2}-q-1$. In particular, since $\mathbb{A}_{5} \cong L_{2}(4)$, if $\mathbb{A}_{5}$ has an $n$-abelian partition, then $n \geqslant 11$. In fact, $\mathbb{A}_{5}$ has a 20-abelian partition, as follows:

$$
\mathbb{A}_{5}=A \uplus A_{1}^{\#} \uplus A_{2}^{\#} \uplus \cdots \uplus A_{20}^{\#},
$$

where $A_{i}^{\#}=A_{i} \backslash\{1\}$, for every $i$, and

$A, A_{1}, \ldots, A_{5}$ are Sylow 5 -subgroups of order 5 ,

$A_{6}, A_{7}, \ldots, A_{15}$ are Sylow 3 -subgroups of order 3 ,

$A_{16}, A_{17}, \ldots, A_{20}$ are Sylow 2-subgroups of order 4 .

Example 2.2. Similarly, if $G_{1}=\mathrm{GL}(2, q)$ and $G_{2}=\mathrm{GL}(3, q)$, $q$ a prime power, then we have

$$
\begin{aligned}
& \left|G_{1}\right|=\left(q^{2}-1\right)\left(q^{2}-q\right) \text { and } \mathrm{k}\left(G_{1}\right)=q^{2}-1, \text { while } \\
& \left|G_{2}\right|=\left(q^{3}-1\right)\left(q^{3}-q\right)\left(q^{3}-q^{2}\right) \text { and } \mathrm{k}\left(G_{2}\right)=q^{3}-q .
\end{aligned}
$$

Again, if $G_{i}$ has an $n_{i}$-abelian partition, for $i=1,2$, by Eq. (1), we obtain $n_{1} \geqslant$ $q(q-1)-1$ and $n_{2} \geqslant q^{2}\left(q^{3}-1\right)(q-1)-1$.

Lemma 2.3. [9, Lemma 4.1] Let $\left\{g_{1}, \ldots, g_{m}\right\}$ be a largest noncommuting subset of $G$. Then $\cap_{i=1}^{m} C_{G}\left(g_{i}\right)$ is an abelian subgroup of $G$.

Proof. Assume the contrary and choose $a, b \in \cap_{i=1}^{m} C_{G}\left(g_{i}\right)$ such that $a b \neq b a$. Then it is easy to see that $\left\{a, b g_{1}, b g_{2}, \ldots, b g_{m}\right\}$ is a noncommuting subset of $G$, a contradiction.

Before stating our main results we introduce another notation. Given a finite group $G$, we denote by $\operatorname{cs}(G)$ the set of conjugacy class sizes of $G$. Itô proved that [7, Theorem 1] if $\operatorname{cs}(G)=\{1, m\}$, then $G$ is a direct product of a Sylow p-group of $G$ with an abelian group. In particular, then $m$ is a power of $p$. 
Theorem 2.4. The following conditions on a nonabelian group $G$ are equivalent:

(1) $G$ has a 2-abelian partition with respect to an abelian subgroup $A$.

(2) $G=P \times Q$, where $P \in \operatorname{Syl}_{2}(G)$ with $P / Z(P) \cong \mathbb{Z}_{2} \times \mathbb{Z}_{2}$ and $Q$ is abelian, and $A=\langle Z(G), t\rangle$, where $t \in G \backslash Z(G)$ and $t^{2} \in Z(G)$.

Proof. (1) $\Rightarrow(2)$ Suppose that $G$ is a nonabelian group, which has a 2-abelian partition $G=A \uplus A_{1} \uplus A_{2}$. First of all, we notice that every noncommuting set of $G$ can have at most three elements. Now fix a noncentral element $x$ of $G$. Since $C_{G}(x)<G$, we can choose $y \in G$, such that $x$ and $y$ do not commute. It is well known that a group cannot be written as the union of two proper subgroups, thus $C_{G}(x) \cup C_{G}(y)<G$, and so we can choose $z$ in $G$, such that $B=\{x, y, z\}$ is a noncommuting set of $G$. Now, we have

$$
G=C_{x} \cup C_{y} \cup C_{z},
$$

where $C_{x}=C_{G}(x), C_{y}=C_{G}(y)$ and $C_{z}=C_{G}(z)$. Put $K=C_{x} \cap C_{y} \cap C_{z}$, which is an abelian subgroup of $G$, by Lemma 2.3. Indeed, by a result of Scorza [10], we have

(a) $\left[G: C_{x}\right]=\left[G: C_{y}\right]=\left[G: C_{z}\right]=2$,

(b) $K=C_{x} \cap C_{y}=C_{x} \cap C_{z}=C_{y} \cap C_{z}$, and

(c) $K$ is a normal subgroup of $G$ and the factor group $G / K$ is isomorphic to the Klein Four Group.

Thus $\left|x^{G}\right|=2$, and since $x \in G \backslash Z(G)$ was arbitrary, it follows that $\operatorname{cs}(G)=\{1,2\}$. By Itô's result [7, Theorem 1], $G=P \times Q$, where $P \in \operatorname{Syl}_{2}(G)$ is nonabelian and $Q \leqslant Z(G)$.

On the other hand, $B$ is a maximal noncommuting set of $G$, which forces $C_{t} \backslash K$ to be a commuting set of $G$ for each $t \in B$, and so the centralizer $C_{t}$ is abelian, because $C_{t}=\left\langle C_{t} \backslash K\right\rangle$. This implies that $K=Z(G)$, and so

$$
\frac{P}{Z(P)} \cong \frac{P \times Q}{Z(P) \times Q}=\frac{G}{Z(G)} \cong \mathbb{Z}_{2} \times \mathbb{Z}_{2},
$$

and the proof is complete.

$(2) \Rightarrow(1)$ Let $\left\{t_{1}, t_{2}, t_{3}, t_{4}\right\}$ be a transversal for $Z(G)$ in $G$, with $t_{1} \in Z(G)$. Then, $G$ is a disjoint union:

$$
G=Z(G) \cup Z(G) t_{2} \cup Z(G) t_{3} \cup Z(G) t_{4} .
$$

Put $A=Z(G) \cup Z(G) t_{2}, A_{1}=Z(G) t_{3}$ and $A_{2}=Z(G) t_{4}$. Then $A$ is an abelian group (since $t_{2}^{2} \in Z(G)$ ), $A_{1}$ and $A_{2}$ are commuting sets, and $G=A \uplus A_{1} \uplus A_{2}$ is a 2-abelian partition of $G$. 
We now work to determine which groups have a 3-abelian partition with respect to an abelian subgroup.

Theorem 2.5. The following conditions on a nonabelian group $G$ are equivalent:

(1) $G$ has a 3-abelian partition with respect to an abelian subgroup $A$.

(2) $|Z(G)| \geqslant 2$ and $G / Z(G)$ is isomorphic to one of the following groups:

$$
\mathbb{Z}_{2} \times \mathbb{Z}_{2}, \mathbb{Z}_{3} \times \mathbb{Z}_{3}, \mathbb{S}_{3}
$$

In the first case, $A=Z(G)$, and in two other cases $A=\langle Z(G), x\rangle$, where $x$ is an element of order 3 outside of $Z(G)$.

Proof. (1) $\Rightarrow(2)$ Suppose that $G$ is a nonabelian group, which has a 3-abelian partition $G=A \uplus A_{1} \uplus A_{2} \uplus A_{3}$. First of all, we notice that $\operatorname{nc}(G)=3$ or 4 . It is now easy to see that $G$ is either 3-centralizer or 4-centralizer, respectively. Therefore, by Theorems 2 and 4 in [1], we conclude that $G$ modulo its center is isomorphic to one of the groups: $\mathbb{Z}_{2} \times \mathbb{Z}_{2}, \mathbb{Z}_{3} \times \mathbb{Z}_{3}$, or $\mathbb{S}_{3}$, as required. Finally, since $G$ is a nonabelian group with at least 7 elements, $|Z(G)| \geqslant 2$.

$(2) \Rightarrow(1)$ Let $Z=Z(G)$. We treat separately the different cases:

(a) $G / Z \cong \mathbb{Z}_{2} \times \mathbb{Z}_{2}$. In this case, there are noncentral elements $x_{1}, x_{2}$, and $x_{3}$ of $G$ such that $G=Z \uplus Z x_{1} \uplus Z x_{2} \uplus Z x_{3}$, which is a 3 -abelian partition of $G$.

(b) $G / Z(G) \cong \mathbb{Z}_{3} \times \mathbb{Z}_{3}$. In this case, we have

$$
G / Z \cong\left\langle Z x, Z y \mid x^{3}, y^{3},[x, y] \in Z\right\rangle,
$$

which implies that

$$
G=Z \cup Z x \cup Z x^{2} \cup Z y \cup Z y^{2} \cup Z x y \cup Z x^{2} y^{2} \cup Z x y^{2} \cup Z x^{2} y .
$$

We put

$$
\begin{aligned}
& A:=Z \cup Z x \cup Z x^{2}=\langle Z, x\rangle, \\
& A_{1}:=Z y \cup Z y^{2}=\langle Z, y\rangle \backslash Z, \\
& A_{2}:=Z x y \cup Z x^{2} y^{2}=\langle Z, x y\rangle \backslash Z, \\
& A_{3}:=Z x y^{2} \cup Z x^{2} y=\left\langle Z, x y^{2}\right\rangle \backslash Z .
\end{aligned}
$$

Then $G=A \uplus A_{1} \uplus A_{2} \uplus A_{3}$ is a 3 -abelian partition of $G$.

(c) $G / Z \cong \mathbb{S}_{3}$. In this case, we have $G / Z \cong\left\langle Z x, Z y \mid x^{3}, y^{2},(x y)^{2} \in Z\right\rangle$, which implies that

$$
G=Z \cup Z x \cup Z x^{2} \cup Z y \cup Z y x \cup Z y x^{2} .
$$


Put $A:=Z \cup Z x \cup Z x^{2}=\langle Z, x\rangle$. Then, $G=A \uplus Z y \uplus Z y x \uplus Z y x^{2}$ is a 3-abelian partition of $G$.

The proof is complete.

Acknowledgements. This work was done during the second author had a visiting position at the Department of Mathematical Sciences, Kent State University, USA. He would like to thank the hospitality of the Department of Mathematical Sciences of KSU and Prof. Mark L. Lewis for useful discussions. This research was also supported by Iran National Science Foundation (INSF: 94028936) while the first author was a Postdoctoral Research Associate at the Faculty of Mathematics of K. N. Toosi University of Technology. The authors would like to thank an anonymous referee for many suggestions that improved the exposition in this paper.

\section{References}

[1] S. M. Belcastro and G. J. Sherman, Counting centralizers in finite groups, Math. Mag., 67(5) (1994), 366-374.

[2] E. A. Bertram, Some applications of graph theory to finite groups, Discrete Math., 44(1) (1983), 31-43.

[3] A. Brandstädt, Partitions of graphs into one or two independent sets and cliques, Discrete Math., 152(1-3) (1996), 47-54.

[4] J. R. Britnell and N. Gill, Perfect commuting graphs, J. Group Theory, 20(1) (2017), 71-102.

[5] A. K. Das and D. Nongsiang, On the genus of the commuting graphs of finite non-abelian groups, Int. Electron. J. Algebra, 19 (2016), 91-109.

[6] S. Földes and P. L. Hammer, Split graphs, Proceedings of the Eighth Southeastern Conference on Combinatorics, Graph Theory and Computing (Louisiana State Univ., Baton Rouge, La., 1977), 311-315.

[7] N. Itô, On finite groups with given conjugate types I, Nagoya Math. J., 6 (1953), $17-28$.

[8] A. Mahmoudifar and A. R. Moghaddamfar, Commuting graphs of groups and related numerical parameters, Comm. Algebra, 45(7) (2017), 3159-3165.

[9] L. Pyber, The number of pairwise noncommuting elements and the index of the centre in a finite group, J. London Math. Soc., 35(2) (1987), 287-295.

[10] G. Scorza, I gruppi che possono pensarsi come somme di tre loro sottogruppi, Boll. Unione Mat. Ital., (1926), 216-218. 


\section{A. Mahmoudifar}

Department of Mathematics

North Tehran Branch

Islamic Azad University

Tehran, Iran

and

Faculty of Mathematics

K. N. Toosi University of Technology

Tehran, Iran

e-mail: alimahmoudifar@gmail.com

A. R. Moghaddamfar (Corresponding Author) and F. Salehzadeh

Faculty of Mathematics

K. N. Toosi University of Technology

Tehran, Iran

e-mails: moghadam@kntu.ac.ir and moghadam@ipm.ir (A.R. Moghaddamfar) salehzadeh.fayez@gmail.com (F. Salehzadeh) 